\title{
Expression Analysis of MISTI and EMT Markers in Primary Tumor Samples Points to MISTI as a Biomarker of Cervical Cancer
}

\author{
Wei Wang' \\ Xin $\mathrm{Xie}^{2}$ \\ Zhangjian Zhou ${ }^{3}$ \\ Hao Zhang (iD) ${ }^{2}$ \\ 'Department of Obstetrics and \\ Gynecology, The First Affiliated Hospital \\ of Xi'an Jiaotong University, Xi'an, \\ Shaanxi, 71006I, People's Republic of \\ China; ${ }^{2}$ Department of Surgical \\ Oncology, The First Affiliated Hospital of \\ Xi'an Jiaotong University, Xi'an, Shaanxi, \\ 7I006I, People's Republic of China; \\ ${ }^{3}$ Department of Surgical Oncology, The \\ Second Affiliated Hospital of Xi'an \\ Jiaotong University, Xi'an, Shaanxi, \\ 71006I, People's Republic of China
}

Correspondence: Hao Zhang Department of Surgical Oncology, The First Affiliated Hospital of Xi'an Jiaotong University, Xi'an, Shaanxi, 7I006I,

People's Republic of China

Tel +8613571946099

Email hao.zhang@mail.xjtu.edu.cn
Background: Mist1 is a basic transcription factor, which plays an important role in the development of multiple organs, and may also regulate tumor progression by mediating epithelial-mesenchymal transformation. However, there is lack of research on its role of squamous cell carcinoma, especially in cervical squamous cell carcinoma.

Methods: Bioinformatic methods were used to analyze gene expression, correlation, and patient survival according to the TCGA database. Thirty pairs of cancer tissues and distal cancer tissues from cervical cancer patients who received radical surgery were enrolled in the study. The expression of Mist1 was analyzed using Western blot. Furthermore, the potential associations among Mist1 expression, EMT biomarkers and various clinicopathological characteristics were investigated. All statistical tests employed in this study were twosided, and $P$ values $<0.05$ were deemed statistically significant.

Results: Overall survival data were obtained from TCGA-CESC dataset, containing 3 control samples and 305 tumor samples. The expression of Mist1 was significantly higher in primary tumor than in normal tissues $(P<0.001)$. The samples were divided into a low Mist1 expression group ( $\mathrm{n}=144)$ and a high Mist1 expression group $(\mathrm{n}=146)$ according to the median expression level. Kaplan-Meier survival analysis revealed that high expression of Mist1 was significantly correlated with poor overall survival $(P=0.032)$. We further explored the relationships between Mist1 and EMT. Among the 30 primary cervical cancer specimens investigated, the difference in Mist1 expressed statuses between cervical cancer tissues and distal noncancerous cervical tissues was significant $(P=0.001)$. And the epithelial cell marker E-cadherin was downregulated in Mist1 overexpressed cervical cancer cells; however, the mesenchymal marker N-Cadherin and Twist was upregulated.

Conclusion: Our study found that Mist1 seemed to play the role of oncogene in cervical squamous cell carcinoma and could be a potential biomarker.

Keywords: Mist1, epithelial-mesenchymal transformation, cervical cancer

\section{Introduction}

Cervical cancer is the fourth most common female malignant tumor in the world, which is a serious threat to the health of women all over the world. According to the Global Cancer Statistics, there were about half million new cases of cervical cancer in 2018. ${ }^{1}$ The incidence and mortality of cervical cancer vary greatly according to the geographical location and economic conditions of different regions. A study of 38 countries on five continents shows that compared with the declining incidence of cervical cancer in economically developed countries, the incidence of cervical 
cancer in economically poor areas is stable or even rising. ${ }^{2}$ The latest national cancer statistics released by the National Cancer Center of China in 2017 show that cervical cancer accounts for $6.25 \%$ of the top 10 female malignant tumors, ranking sixth. ${ }^{3}$ Mist1, also known as bHLHa15, is a member of the helix-loop-helix (basic helix-loop-helix, bHLH) protein family. ${ }^{4}$ This protein family is a basic transcription factor, which regulates target genes by forming homologous or heterodimers that bind to the E-box sequence of target genes. In previous studies, Mist1 plays an important role in the development of multiple organs, and may also regulate tumor progression by mediating epithelial-mesenchymal transformation in the occurrence and development of pancreatic tumor and gastric cancer. ${ }^{5-8}$ However, there is a lack of research on the role of squamous cell carcinoma, especially in cervical squamous cell carcinoma. Therefore, through the different expression levels of Mist1 in cervical cancer, this paper attempts to explore its role in cervical cancer.

\section{Materials and Methods}

\section{Gene Screening}

GEPIA (Interactive Analysis of Gene Expression Analysis, http://gepia.cancer-pku.cn/) and UALCAN (http://ualcan.path.uab.edu/index.html), the web-based tools were used to analyze gene expression, correlation analysis, and patient survival analysis according to the cancer genome atlas (TCGA) database. ${ }^{9,10}$ We analyzed the expression of Mist1 and its relationship with overall survival in cervical squamous cell carcinoma and endocervical adenocarcinoma (CESC) dataset. For survival analysis, the median expression of transcripts per kilobase million (TPM) was selected as the threshold for splitting the high-expression and low-expression cohorts. Samples with expression level higher than this threshold are considered as the high-expression cohort and vice versa. At the meanwhile, we analyzed the relationship between Mist1 expression and clinical characteristics including age, race, weight, lymph node metastasis, and tumor stage. Further, the relationship between Mist1 and other correlated pathway factors was calculated.

GeneMANIA (http://www.genemania.org) is a webbased tool was used to build protein-protein interaction (PPI) networks, generating hypotheses about gene function, analyzing gene lists and prioritizing them. We visualized the physical interaction gene network and then highlighted the pathway using GeneMANIA. ${ }^{11,12}$

\section{Patients and Tissues}

Thirty pairs of cancer tissues and distal noncancerous tissues from cervical cancer patients who received radical surgery were enrolled in the study at the First Affiliated Hospital of Xi'an Jiaotong University from July 2019 to April 2020. This study was complied with the Helsinki Declaration and approved by the Ethics Committee of the First Affiliated Hospital of Xi'an Jiaotong University. All patients provided their written informed consents. All patients did not receive any chemotherapy or radiotherapy before surgery.

\section{RNA Extraction and Quantitative Real-Time PCR (qPCR)}

All tissue specimens' RNA was extracted using TRIzol reagent following the manufacturer's protocol (Invitrogen, USA). Reversed transcription was completed using the PrimeScript ${ }^{\mathrm{TM}}$ RT reagent Kit (Takara, Japan) and qPCR was performed on Bio-Rad ${ }^{\circledR}$ CFX96 Touch $^{\text {TM }}$ Real-Time PCR Detection System using SYBR Premix Ex TaqTM (Takara, Japan) with the qPCR conditions as follows: Step $195^{\circ} \mathrm{C}$ for $30 \mathrm{~s}$; Step $295^{\circ} \mathrm{C}$ for $5 \mathrm{~s}$ and $60^{\circ} \mathrm{C}$ for 30 s for 40 cycles. The following primers were used for qPCR amplification: Mist1: forward, 5'-CGGATGCAC AAGCTAAATAACG-3'; reverse, 5'-GCCGT CAGCGATTTGATGTAG-3'. GAPDH: forward, 5'- GTC AAGGCTGAGAACGGGAA-3'; reverse, 5'- AAATGAG CCCCAGCCTTCTC-3'. The relative mRNA level was expressed as the fold change relative to the GAPDH gene and normalized to the control group.

\section{Western Blotting}

Cells were lysed on ice with RIPA buffer with 1\% PMSF and $2 \%$ protease inhibitor cocktails (CW Bio, China). The concentration of protein was quantified by the bicinchoninic acid assay (CW Bio, China). Protein samples were separated on $10 \%$ sodium dodecyl sulfate-polyacrylamide gel, electrophoresed and then transferred to polyvinylidene difluoride membrane. The membrane was blocked with $5 \%$ fat free milk at room temperature for $2 \mathrm{~h}$ and incubated overnight at $4^{\circ} \mathrm{C}$ with primary antibody targeting Mist1, E-cadherin, N-cadherin, Snail, Twist1, $\beta$-catenin, TCF4, c-Myc, cyclin D1 and GAPDH (CST, USA), then following by incubation with secondary antibody (Protein Tech, China) at room temperature for $1.5 \mathrm{~h}$. Immunoreactivity was detected by electrochemiluminescence system (Millipore, Germany). 


\section{Statistical Analysis}

Continuous data were expressed as the mean \pm standard deviation. Categorical variables were clustered and compared by the $\chi 2$ test or Fisher's exact test. Continuous variables were compared by the Student's $t$-test. Overall survival and diseases free survival (DFS) were estimated using the Kaplan-Meier method and Log rank test. All statistical tests employed in this study were two-sided, and $P$ values $<0.05$ were deemed statistically significant. Statistical analyses were conducted with $\mathrm{R}$ software version 3.6.2 (http://www.rproject.org).

\section{Results}

To detect the role of Mist1 in cervical carcinoma, we first analyzed the survival rate and gene expression in patients with cervical carcinoma. Overall survival data were obtained from TCGA-CESC dataset, containing three control samples (median TPM 0.094, ranged 0-0.175) and 305 tumor samples (median TPM 0.371, ranged 0-2.352). The expression of Mist1 was significantly higher in primary tumor than in normal tissues $(P<0.001$, Figure 1A). The samples were divided into a low Mist1 expression group $(\mathrm{n}=144)$ and a high Mist1
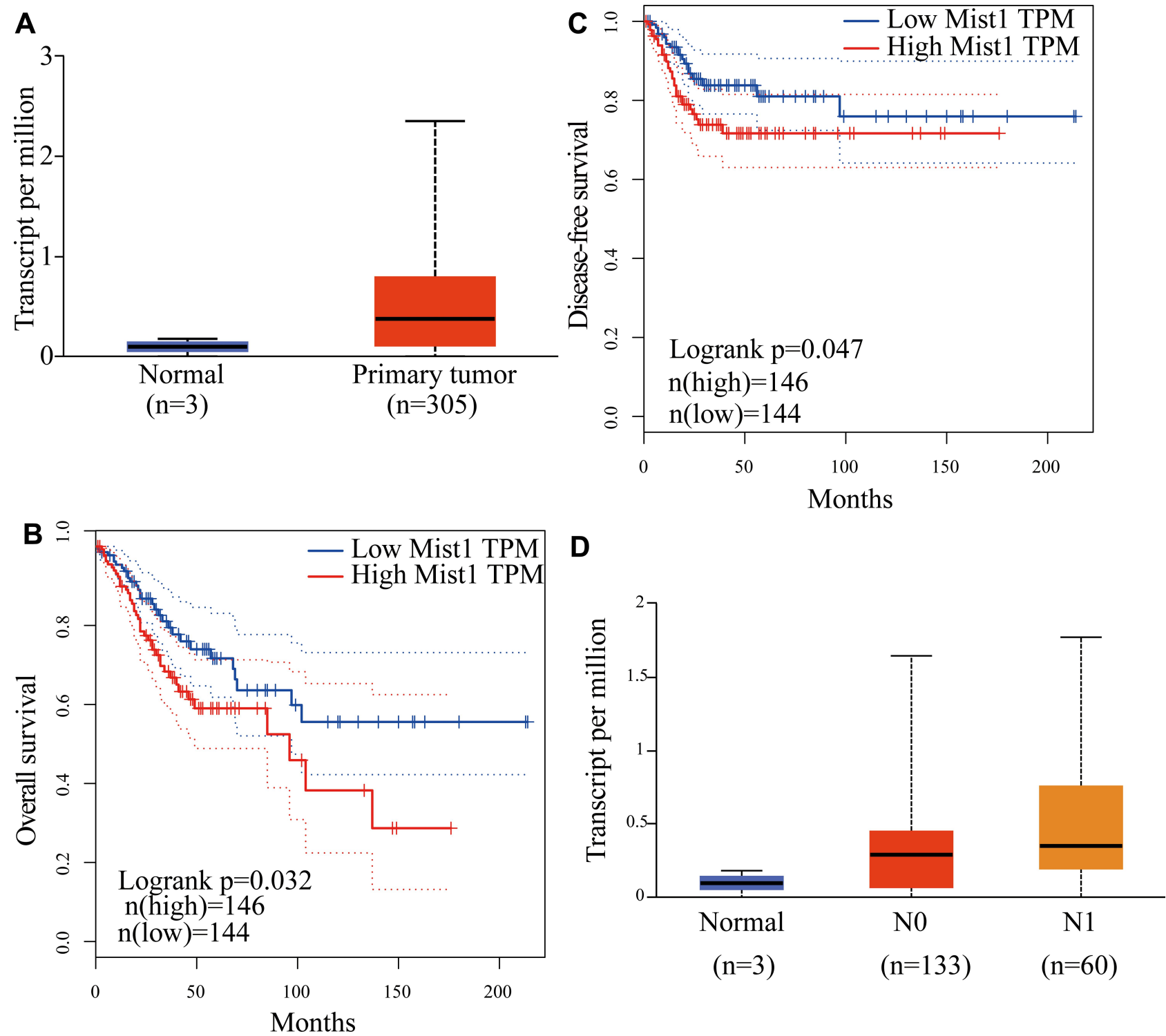

Figure I The relationship between Mist I expression and clinical pathology of cervical cancer. (A) Boxplot showing relative expression of Mist I in tumor and normal tissues from TCGA-CESC patients via UALCAN. (B) Impact of Mistl expression on overall survival in TCGA-CESC patients. (C) Impact of Mistl expression on disease-free survival in TCGA-CESC patients. (D) Boxplot showing relative expression of Mistl in nodal metastasis status (Normal, N0 or NI) from normal and CESC patients via UALCAN. 
expression group $(n=146)$ according to the median expression level. Kaplan-Meier survival analysis revealed that high expression of Mist1 was significantly correlated with poor overall survival $(P=0.032$, Figure $1 \mathrm{~B})$ and diseasefree survival $(P=0.047$, Figure $1 C)$. Further, the expression of Mist1 in different lymph node metastasis groups was analyzed, and there was an increased trend of Mist1 expression in patients with metastatic lymph nodes (Figure 1D).

Next, we analyzed the correlation between these coexpressed genes with Mist1 in UALCAN (the heatmap is shown in Figure 2A) and the EMT related genes were analyzed in GEPIA (Figure 2B). As a transcriptional factor, Mist1 always functions by binding to another protein. Through proteome analysis, we found 20 interacting proteins of Mist1 and displayed them in a physical interaction network using GeneMANIA (Figure 2C). The five most reliable proteins were TCF3, MYOD1, TCF4, GHRHR and SLC22A12.

Due to the fact that high expression of Mist1 involved poor survival of patients with cervical carcinomas. We further explored the relationships between Mist1 and EMT, which played a critical role in tumorigenesis and metastasis. The 30 cervical squamous cancer patients were aged from 30 to 78 years (mean age, 54.53 years). Based on the AJCC staging system, 25 tumors were stage I, II and 5 were Stage III. The details of other clinicopathological characteristics of cervical cancer patients are shown in Table 1 . Among the 30 primary cervical cancer specimens investigated, 23 showed Mist1 overexpressed (Figure 3). The difference in Mist1 expressed statuses between cervical cancer tissues and distal noncancerous cervical tissues was significant $(P=0.001)$.

Notably, the epithelial cell marker E-cadherin was downregulated in Mist1 overexpressed cervical cancer cells; however, the mesenchymal marker N-cadherin and Twist was upregulated (Figure 4). Previous studies demonstrated that Mist1 plays an important role in Wnt/ $\beta$-catenin signaling pathway in carcinogenesis, metastasis and EMT, among which $\beta$-catenin is the core molecule of canonical Wnt $\beta$-catenin pathway, which regulates activities of many downstream signals. In our study, we explored the expression of Wnt $\beta$-catenin signaling pathway in Mist1 expressed cervical cancer tissues and found that $\beta$ catenin was upregulated in Mist1 overexpressed cancer tissues (Figure 4). Then, we checked downstream signals of $\beta$-catenin and found TCF-4, c-Myc and cyclin D1 were also increased in Mist1 over expressed cancer tissues (Figure 4).

\section{Discussion}

The occurrence of cervical cancer is a relatively long process. It takes about 5-10 years to evolve from cervical intraepithelial neoplasia to early invasive cancer, which involves the changes of multiple genes. It is very important to choose appropriate cervical cancer screening methods if these genes can be identified in time and targeted treatment of cervical precancerous lesions can block the progression of cervical epithelial carcinoma and prevent further carcinogenesis. ${ }^{13}$ At present, the screening methods of cervical cancer include thin prep cytologic test, HPV-DNA detection, and cervical biopsy. ${ }^{14,15}$ In spite of the HPV screening and predictive method, the sensitivity and specificity of others are still low.

Through our bioinformatics analysis, we found that the expression of Mist1 was increased in patients with cervical cancer, and its high expression was closely related to the overall survival time of patients with cervical cancer. In addition, our previous study found that Mist1 was related to the expression of E-cadherin and Snail, suggesting that Mist1 may be related to epithelial-mesenchymal transformation. In patients with pancreatic cancer, colorectal cancer, gastric cancer and other tumors, Mist1 can activate Wnt/ $\beta$-Catenin signal pathway, and ultimately promote the development of tumors. ${ }^{16-18}$

In the process of tumor development of digestive organs, a variety of transcription factors that maintain the stable state of normal cells are inhibited. In gastric and pancreatic cancer cells, the restoration of expression of these transcription factors has been proved to effectively reduce the invasiveness and migration of gastric cancer and pancreatic cancer cells, such as SPG20, GATA6, PTF1A, E47. ${ }^{19-21}$ At present, many studies have shown that the loss of Mist1 expression may be an early event in the development of gastric and pancreatic cancer, and its expression plays a role in tumor suppressor genes. ${ }^{22}$ But outside of adenocarcinoma, Mist1 seems to play a different role in squamous cell carcinoma. Mist1 plays the role of oncogenes in squamous cell carcinoma, and its high expression may promote the occurrence and development of squamous cell carcinoma. ${ }^{23}$

At present, there are 19 known members of the Wnt gene family, which affect the biological behavior of cells, such as cell proliferation, differentiation and tumorigenesis. Although different Wnts have their own functions, 


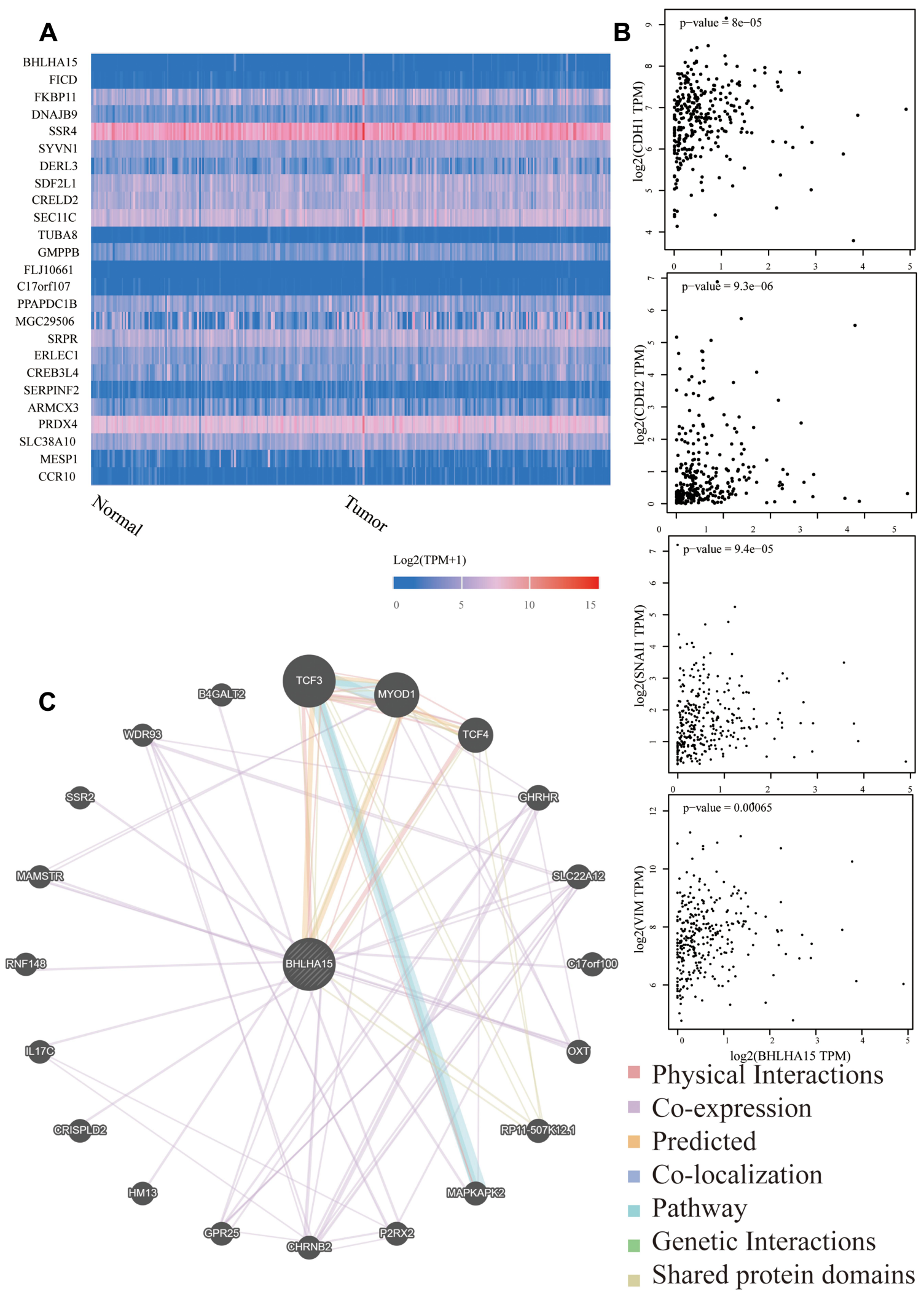

Figure 2 Functional annotations of Mistl co-expressed genes and interaction proteins in TCGA-CESC. (A) Heat maps showing genes positively correlated with Mistl in TCGA-CESC. (B) The scatter plot shows Pearson correlation of Mistl expression with expression E-cadherin, $\mathrm{N}$-cadherin, Snail and Vimentin in TCGA-CESC patients. (C) Protein-protein interaction network of Mistl (GeneMANIA). 
Table I The Demographic and Clinicopathological Characteristics of Patients with Cervical Cancer

\begin{tabular}{|c|c|c|c|}
\hline & Mist I Overexpressed & Mist I Not Overexpressed & $P$ value \\
\hline Age (mean $\pm S D)$ & $54.43 \pm 11.05$ & $54.86 \pm 13.26$ & 0.933 \\
\hline \multicolumn{4}{|l|}{ Stage (n) } \\
\hline Stage I & 12 & 3 & \\
\hline Stage II & 7 & 3 & \\
\hline Stage III & 4 & 1 & 0.83 \\
\hline \multicolumn{4}{|l|}{ Histology (n) } \\
\hline GI & 11 & 2 & \\
\hline G2 & 7 & 5 & \\
\hline G3 & 5 & 0 & 0.12 \\
\hline Size $(\mathrm{mm})$ & $28.00 \pm 13.43$ & $41.00 \pm 24.13$ & 0.076 \\
\hline \multicolumn{4}{|c|}{ Lymph node invasion (n) } \\
\hline With & 6 & 2 & \\
\hline Without & 17 & 5 & 0.896 \\
\hline
\end{tabular}

they also have the same effect. According to a large number of studies, abnormal activation of Wnt plays a role in the formation of a variety of tumors, including colorectal cancer, head and neck malignant tumors, malignant melanoma and so on. ${ }^{24}$ In normal cells, $\beta$-catenin can bind to E-cadherin and regulate the adhesion between homologous cells. In addition, it can also act on APC gene, glycogen synthesis kinase-3 $\beta$ and cytoskeletal proteins to form a polyprotein complex in vivo, and then degrade, so that the free $\beta$-catenin in the cytoplasm is always kept at a low state. Due to the existence of gene mutations, such as APC gene or $\beta$-catenin gene, the degradation of $\beta$-catenin will be hindered and concentrated in the cytoplasm, at the same time, transfer to the nucleus, and combined with the transcription factor Tcf/Lef, resulting in excessive cell proliferation or the invasiveness. If the $\mathrm{Wnt} / \beta$-catenin signal pathway has been in an abnormal state, a large number of $\beta$-catenin proteins in the cytoplasm will gather and bind to Tcf/Lef at the nuclear location, so that the related transcription process is activated, which leads to the occurrence of cancer. There is evidence that $85 \%$ of the patients with sporadic and hereditary colon cancer have lost the normal function of APC gene, while colon cancer cells have mutated APC gene. ${ }^{25}$ The reason why APC gene can inhibit tumor is to regulate the level of $\beta$-catenin in cells. Another research observed the expression level of $\beta$ catenin in a study of ovarian cancer, and the results showed that the abnormal expression rate of $\beta$-catenin in ovarian cancer was much higher than that in benign ovarian tumors. Under microscope, the expression of $\beta$-catenin was decreased or even not expressed in the membrane and cytoplasm. The expression characteristics of these

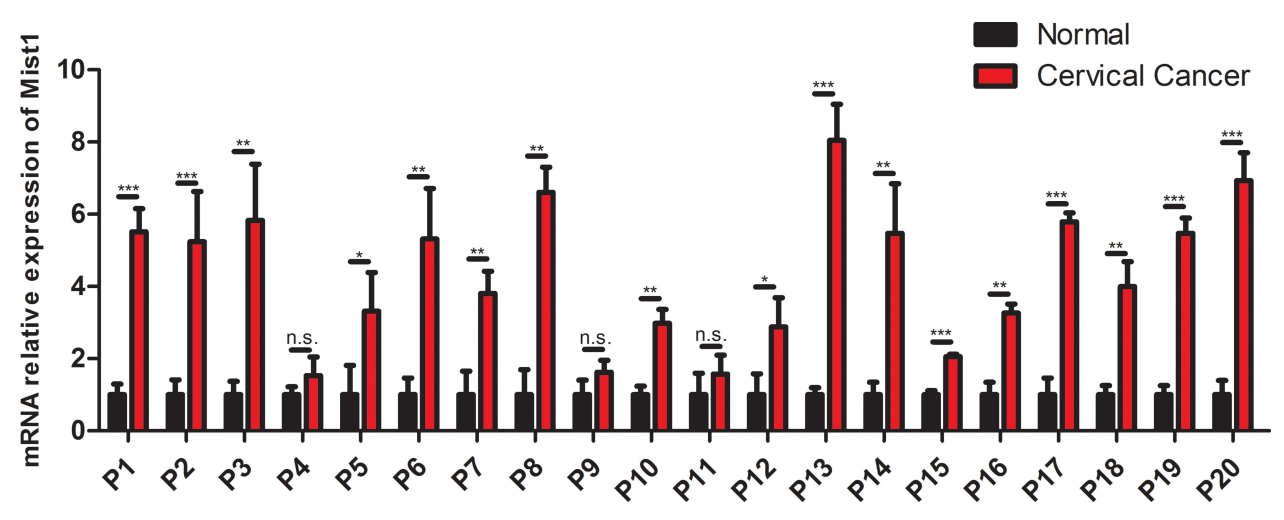

Figure 3 The Mistl expression in cancer and distal noncancerous tissues of patients with cervical cancer. $* P<0.05, * * P<0.0$, $* * * P<0.001$. Abbreviation: n.s.: no significant difference. 
A

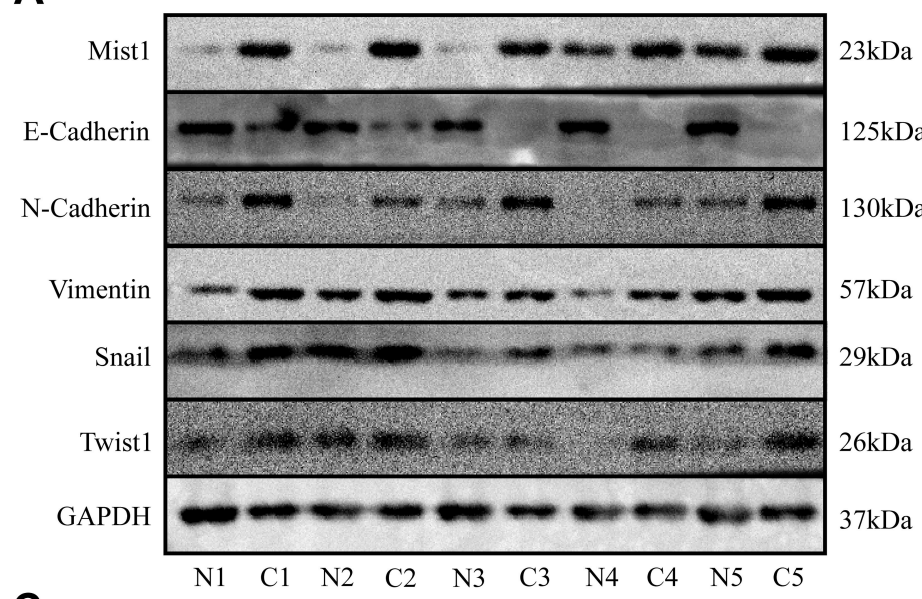

C

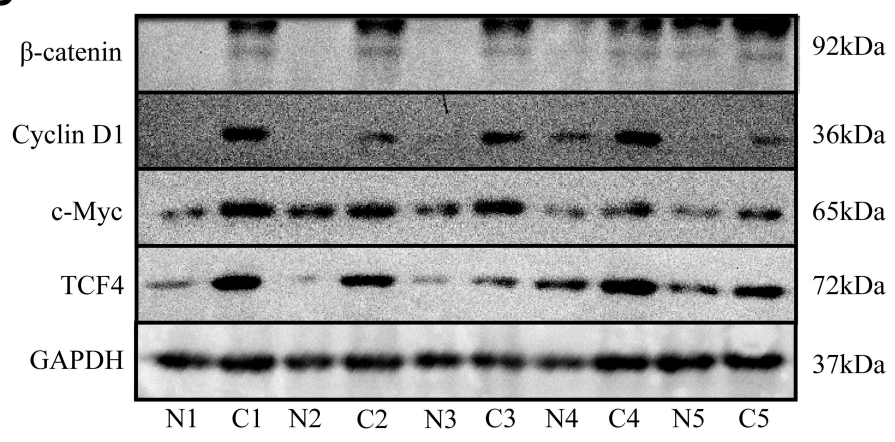

B

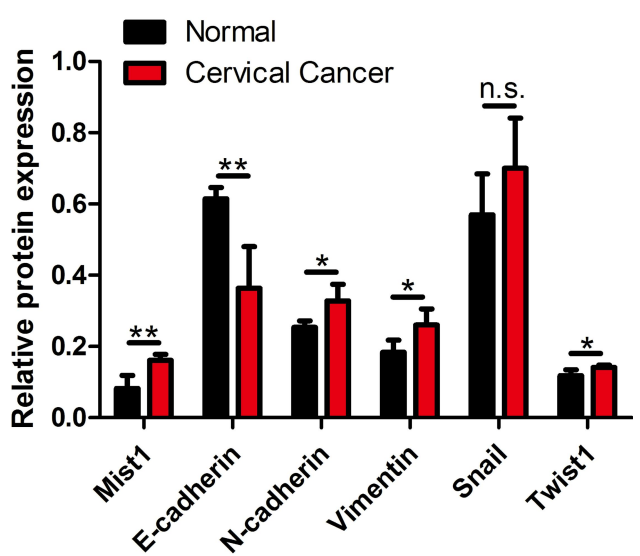

D

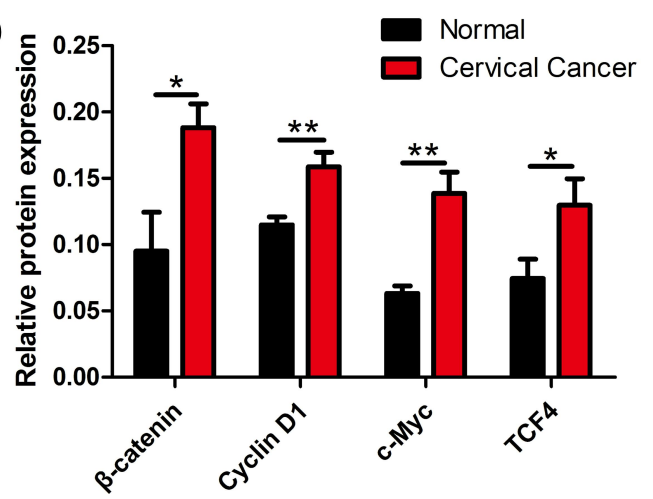

Figure 4 The relationship between Mistl and EMT markers and Wnt signaling pathway in patients with cervical cancer. (A) The protein expression of Mistl, E-cadherin, $\mathrm{N}$-cadherin, Snail, Vimentin and Twist was measured by the Western blot analysis. (B) Quantification of Mistl, E-cadherin, N-cadherin, Snail, Vimentin and Twist protein expression in patients with cervical cancer. (C) The protein expression of $\beta$-catenin, cyclin DI, c-Myc and TCF4 was measured by the Western blot analysis. (D) Quantification of $\beta$-catenin, cyclin DI, c-Myc and TCF4 protein expression in patients with cervical cancer. $* P<0.05, * * P<0.01$.

Abbreviation: n.s., no significant difference.

expressions show that there is a certain relationship between $W n t / \beta$-catenin pathway and the occurrence and development of variety kinds of cancers. ${ }^{26}$

The study has some limitations. First, the occurrence of cervical cancer is closely related to HPV infection, and the pathogenesis of cervical cancer in different HPV infection states is also different. Regrettably, our study did not clarify the pathogenesis of Mist1 in different HPV infection states, which also requires further research. Second, only three normal specimens can be obtained from the TCGA public data. This will cause a certain bias of the analysis.

To conclude, our study found that Mist1 seemed to play the role of oncogene in cervical squamous cell carcinoma and could be a potential biomarker. This is different from the previous expression of Mist1 in other adenocarcinomas, so its role in tumorigenesis remains to be further studied.

\section{Ethics}

This investigation was approved by the Ethics Committee of the First Affiliated Hospital of Xi'an Jiaotong University.

\section{Author Contributions}

All authors made substantial contributions to conception and design, acquisition of data, or analysis and interpretation of data; took part in drafting the article or revising it critically for important intellectual content; agreed to submit to the current journal; gave final approval of the version to be published; and agree to be accountable for all aspects of the work.

\section{Funding}

This study was supported by the National Natural Science Foundation of China (No. 81502442).

\section{Disclosure}

The authors report no conflicts of interest in this work. 


\section{References}

1. Bray F, Ferlay J, Soerjomataram I, Siegel R, Torre L, Jemal A. Global cancer statistics 2018: GLOBOCAN estimates of incidence and mortality worldwide for 36 cancers in 185 countries. CA Cancer J Clin. 2018;68(6):394-424. doi:10.3322/caac.21492

2. Vaccarella S, Lortet-Tieulent J, Plummer M, Franceschi S, Bray F. Worldwide trends in cervical cancer incidence: impact of screening against changes in disease risk factors. Eur J Cancer. 2013;49 (15):3262-3273. doi:10.1016/j.ejca.2013.04.024

3. Chen W, Zheng R, Baade P, et al. Cancer statistics in China, 2015. CA Cancer J Clin. 2016;66(2):115-132. doi:10.3322/caac.21338

4. Morris J, Wang S, Hebrok M. KRAS, Hedgehog, Wnt and the twisted developmental biology of pancreatic ductal adenocarcinoma. Nat Rev Cancer. 2010;10(10):683-695. doi:10.1038/nrc2899

5. Yamaguchi J, Mino-Kenudson M, Liss A, et al. Loss of Trefoil Factor 2 from pancreatic duct glands promotes formation of intraductal papillary mucinous neoplasms in mice. Gastroenterology. 2016;151 (6):1232-44.e10. doi:10.1053/j.gastro.2016.07.045

6. Riera K, Jang B, Min J, et al. Trop2 is upregulated in the transition to dysplasia in the metaplastic gastric mucosa. J Pathol. 2020;251 (3):336-347. doi:10.1002/path.5469

7. Alison M. The cellular origins of cancer with particular reference to the gastrointestinal tract. Int J Exp Pathol. 2020;101:132-151. doi:10.1111/iep.12364

8. Hsieh M, Jeng Y, Lee Y. Mist1: a novel nuclear marker for acinic cell carcinoma of the salivary gland. Virchows Archiv Int $J$ Pathol. 2019;475(5):617-624. doi:10.1007/s00428-019-02600-1

9. Chandrashekar D, Bashel B, Balasubramanya S, et al. UALCAN: a portal for facilitating tumor subgroup gene expression and survival analyses. Neoplasia (New York, NY). 2017;19(8):649-658. doi:10.1016/j.neo.2017.05.002

10. Tang Z, Li C, Kang B, Gao G, Li C, Zhang Z. GEPIA: a web server for cancer and normal gene expression profiling and interactive analyses. Nucleic Acids Res. 2017;45:W98-W102. doi:10.1093/nar/gkx247

11. Bai M, Che Y, Lu K, Fu L. Analysis of deubiquitinase OTUD5 as a biomarker and therapeutic target for cervical cancer by bioinformatic analysis. PeerJ. 2020;8:e9146. doi:10.7717/peerj.9146

12. Warde-Farley D, Donaldson S, Comes O, et al. The GeneMANIA prediction server: biological network integration for gene prioritization and predicting gene function. Nucleic Acids Res. 2010;38:W21420. doi:10.1093/nar/gkq537

13. Mejía L, Muñoz D, Trueba G, Tinoco L, Zapata S. Prevalence of human papillomavirus types in cervical cancerous and precancerous lesions of Ecuadorian women. J Med Virol. 2016;88(1):144-152. doi:10.1002/jmv.24310

14. Kori M, Yalcin Arga K. Potential biomarkers and therapeutic targets in cervical cancer: insights from the meta-analysis of transcriptomics data within network biomedicine perspective. PLoS One. 2018;13(7): e0200717. doi:10.1371/journal.pone.0200717
15. Litjens R, Hopman A, van de Vijver K, Ramaekers F, Kruitwagen R, Kruse A. Molecular biomarkers in cervical cancer diagnosis: a critical appraisal. Expert Opin Med Diagn. 2013;7(4):365-377. doi: $10.1517 / 17530059.2013 .808621$

16. Nienhüser H, Kim W, Malagola E, et al. Mist1+ gastric isthmus stem cells are regulated by Wnt5a and expand in response to injury and inflammation in mice. Gut. 2020;70:654-665. doi:10.1136/gutjnl2020-320742

17. Yamada S, Yamanoi K, Sato Y, Nakayama J. Diffuse MIST1 expression and decreased $\alpha 1$, 4-linked N-acetylglucosamine ( $\alpha \mathrm{GlcNAc}$ ) glycosylation on MUC6 are distinct hallmarks for gastric neoplasms showing oxyntic gland differentiation. Histopathology. 2020;77 (3):413-422. doi:10.1111/his.14165

18. Young C, Baker R, Howlett C, et al. The loss of ATRX increases susceptibility to pancreatic injury and oncogenic KRAS in female but not male mice. Cell Mol Gastroenterol Hepatol. 2019;7(1):93-113. doi:10.1016/j.jemgh.2018.09.004

19. Brembeck F, Schreiber F, Deramaudt T, et al. The mutant K-ras oncogene causes pancreatic periductal lymphocytic infiltration and gastric mucous neck cell hyperplasia in transgenic mice. Cancer Res. 2003;63(9):2005-2009.

20. Guerra C, Schuhmacher A, Cañamero M, et al. Chronic pancreatitis is essential for induction of pancreatic ductal adenocarcinoma by K-Ras oncogenes in adult mice. Cancer Cell. 2007;11(3):291-302.

21. Zhou Z, Wang W, Xie X, Song Y, Dang C, Zhang H. Methylationinduced silencing of SPG20 facilitates gastric cancer cell proliferation by activating the EGFR/MAPK pathway. Biochem Biophys Res Commun. 2018;500(2):411-417. doi:10.1016/j.bbrc.2018.04.089

22. Direnzo D, Hess D, Damsz B, et al. Induced Mist1 expression promotes remodeling of mouse pancreatic acinar cells. Gastroenterology. 2012;143(2):469-480. doi:10.1053/j. gastro.2012.04.011

23. Chung W, Zhou Y, Atfi A, Xu K. Downregulation of notch signaling in Kras-induced gastric metaplasia. Neoplasia (New York, NY). 2019;21(8):810-821. doi:10.1016/j.neo.2019.06.003

24. Clevers H, Nusse R. Wnt/ $\beta$-catenin signaling and disease. Cell. 2012;149(6):1192-1205. doi:10.1016/j.cell.2012.05.012

25. Sakai E, Nakayama M, Oshima H, et al. Combined mutation of Apc, Kras, and Tgfbr2 effectively drives metastasis of intestinal cancer. Cancer Res. 2018;78(5):1334-1346. doi:10.1158/0008-5472.CAN17-3303

26. Nguyen V, Hough R, Bernaudo S, Peng C. Wnt/ $\beta$-catenin signalling in ovarian cancer: insights into its hyperactivation and function in tumorigenesis. J Ovarian Res. 2019;12(1):122. doi:10.1186/s13048019-0596-z
International Journal of General Medicine

\section{Publish your work in this journal}

The International Journal of General Medicine is an international, peer-reviewed open-access journal that focuses on general and internal medicine, pathogenesis, epidemiology, diagnosis, monitoring and treatment protocols. The journal is characterized by the rapid reporting of reviews, original research and clinical studies across all disease areas. The manuscript management system is completely online and includes a very quick and fair peer-review system, which is all easy to use. Visit http://www.dovepress.com/ testimonials.php to read real quotes from published authors. 